\title{
Teaching and learning materials on the quasi-steady-state approximation and the partial-equilibrium approximation
}

Agnès Pellissier-Tanon*1,2, Gabriel Morgado ${ }^{1}$, Ludovic Jullien ${ }^{2}$, and Annie Lemarchand*1

${ }^{1}$ Laboratoire de Physique Théorique de la Matière Condensée, Sorbonne Université, CNRS , 4 place Jussieu, case courrier 121, 75252 Paris CEDEX 05, France

${ }^{2}$ Laboratoire PASTEUR, Département de chimie, École normale supérieure, PSL University, Sorbonne Université, CNRS, 24 rue Lhomond, 75005 Paris, France

Two approximations exploiting evolutions at different time scales are usually used to simplify rate laws in chemical kinetics. ${ }^{1-5}$ The quasi-steady-state approximation (QSSA) is used to eliminate a chemical species which evolves faster than the others. ${ }^{6}$ The partialequilibrium approximation (PEA) is employed to remove a reaction step associated with an extent evolving faster than the other extents. Both methods are examples of adiabatic elimination of a fast variable in differential equations. ${ }^{7-16}$ The fast variable, concentration or extent, is supposed to instantaneously adapt to the evolution of the other variables. For this reason, the fast variable is sometimes called a slave variable. ${ }^{17}$ The fast variable is not independent from the other variables and can be ignored in the reduced rate equations. Quasi-steady state or partial equilibrium does not mean that the eliminated variable has reached a steady state but that it keeps evolving at a rate imposed by the other variables. Far from being stationary, the evolution of the eliminated variable occurs at the time scale on which the reduced rate equations focus.

\section{Simple example of quasi-steady-state approxima- tion}

The first-order reaction scheme

$$
\mathrm{X} \stackrel{k_{1}}{\longrightarrow} \mathrm{Y} \stackrel{k_{2}}{\longrightarrow} \mathrm{Z}
$$


is associated with the rate equations

$$
\begin{aligned}
& \frac{\mathrm{d} X}{\mathrm{~d} t}=-k_{1} X \\
& \frac{\mathrm{d} Y}{\mathrm{~d} t}=k_{1} X-k_{2} Y
\end{aligned}
$$

that can be easily solved leading to

$$
\begin{aligned}
& X(t)=X(0) \exp \left(-k_{1} t\right) \\
& Y(t)=\frac{k_{1} X(0)}{k_{2}-k_{1}} \exp \left(-k_{1} t\right)+\left(Y(0)-\frac{k_{1} X(0)}{k_{2}-k_{1}}\right) \exp \left(-k_{2} t\right)
\end{aligned}
$$

If the rate constant $k_{1}$ associated with the formation of species $\mathrm{Y}$ is much smaller than the rate constant $k_{2}$ associated with the consumption of $\mathrm{Y}$, the concentration $Y$ possesses an exponential component $\exp \left(-k_{2} t\right)$ evolving faster than the concentration $X$ which evolves as $\exp \left(-k_{1} t\right)$. At a time scale larger than $1 / k_{2}$, the fast exponential component of $Y$ is negligible leading to

$$
\begin{aligned}
& X(t)=X(0) \exp \left(-k_{1} t\right) \\
& Y(t) \simeq \frac{k_{1} X(0)}{k_{2}} \exp \left(-k_{1} t\right)
\end{aligned}
$$

where $k_{2}-k_{1} \simeq k_{2}$ has been used. Equations $(6,7)$ imply

$$
Y(t) \simeq \frac{k_{1}}{k_{2}} X(t)
$$

which is straightforwardly deduced from Eq. (3) assuming $\frac{\mathrm{d} Y}{\mathrm{~d} t}=0$. Hence, the quasisteady-state approximation does not impose that $Y$ has reached a steady-state for which $\frac{\mathrm{d} X}{\mathrm{~d} t}=0$ would be also necessary. According to Eq. (8), the approximation imposes that $Y$ follows the evolution of $X$.

The evolution of the concentration $X$ given in Eq. (6) can be associated with the reduced reaction scheme

$$
\mathrm{X} \stackrel{k_{1}}{\rightarrow} \mathrm{Z}
$$

in which the fast variable $\mathrm{Y}$ has been eliminated. In this simple example with a reactive intermediate $\mathrm{Y}$, the concentration $Y$ is smaller than $X$, which does not provide a general validity condition for the elimination of $Y$ using QSSA. The application of the approximation requires a condition on the time scales characterizing the evolution of the concentrations. In this simple example, the condition is $k_{1} \ll k_{2}$. 


\section{Simple example of partial-equilibrium approxima- tion}

The first-order reaction scheme

$$
\mathrm{X} \underset{k_{-1}}{\stackrel{k_{1}}{\rightleftharpoons}} \mathrm{Y} \stackrel{k_{2}}{\longrightarrow} \mathrm{Z}
$$

with rate constants obeying $k_{1} \gg k_{2}$ and $k_{-1} \gg k_{2}$ is associated with the following rate equations for the extents of reaction $\xi_{1}$ and $\xi_{2}$ associated with the first and second steps

$$
\begin{aligned}
& \frac{\mathrm{d} \xi_{1}}{\mathrm{~d} t}=k_{1} X-k_{-1} Y \\
& \frac{\mathrm{d} \xi_{2}}{\mathrm{~d} t}=k_{2} Y
\end{aligned}
$$

where $X$ and $Y$ are the concentrations of species $\mathrm{X}$ and $\mathrm{Y}$. The rate equations for the concentrations are

$$
\begin{aligned}
\frac{\mathrm{d} X}{\mathrm{~d} t} & =-k_{1} X+k_{-1} Y \\
\frac{\mathrm{d} Y}{\mathrm{~d} t} & =k_{1} X-\left(k_{-1}+k_{2}\right) Y \\
\frac{\mathrm{d} Z}{\mathrm{~d} t} & =k_{2} Y
\end{aligned}
$$

leading to

$$
\begin{aligned}
\frac{\mathrm{d} \xi_{1}}{\mathrm{~d} t} & =-\frac{\mathrm{d} X}{\mathrm{~d} t} \\
\frac{\mathrm{d} \xi_{2}}{\mathrm{~d} t} & =\frac{\mathrm{d} Z}{\mathrm{~d} t}
\end{aligned}
$$

The law of conservation of matter imposes

$$
X+Y+Z=C_{\text {tot }}
$$

where $C_{\text {tot }}$ is the total concentration. For vanishing extents at the steady state, $\left(X_{0}, Y_{0}, Z_{0}\right)=$ $\left(0,0, C_{\text {tot }}\right)$, the relationships between the concentrations and the extents are deduced from the integration of Eqs. $(16,17)$ leading to

$$
\begin{aligned}
& \xi_{1}=-X \\
& \xi_{2}=-X-Y
\end{aligned}
$$

Eqs. $(11,12,19,20)$ are used to write the rate equations for the extents:

$$
\begin{aligned}
& \frac{\mathrm{d} \xi_{1}}{\mathrm{~d} t}=-\left(k_{1}+k_{-1}\right) \xi_{1}+k_{-1} \xi_{2} \\
& \frac{\mathrm{d} \xi_{2}}{\mathrm{~d} t}=k_{2} \xi_{1}-k_{2} \xi_{2}
\end{aligned}
$$


that can be solved using standard linear algebra calculations. For $k_{1} \gg k_{2}$ and $k_{-1} \gg k_{2}$, it reads

$$
\begin{aligned}
& \xi_{1}(t) \simeq \frac{k_{-1}}{\sqrt{\left(k_{1}+k_{-1}\right)^{2}+k_{-1}^{2}}} A(0) \exp \left(-\frac{k_{1} k_{2}}{k_{1}+k_{-1}} t\right)-B(0) \exp \left(-\left(k_{1}+k_{-1}\right) t\right) \\
& \xi_{2}(t) \simeq \frac{k_{1}+k_{-1}}{\sqrt{\left(k_{1}+k_{-1}\right)^{2}+k_{-1}^{2}}} A(0) \exp \left(-\frac{k_{1} k_{2}}{k_{1}+k_{-1}} t\right)+\frac{k_{2}}{k_{1}+k_{-1}} B(0) \exp \left(-\left(k_{1}+k_{-1}\right) t\right)
\end{aligned}
$$

with

$$
\begin{aligned}
& A(0)=\frac{k_{2} \sqrt{\left(k_{1}+k_{-1}\right)^{2}+k_{-1}^{2}}}{\left(k_{1}+k_{-1}\right)^{2}} \xi_{1}(0)+\frac{\sqrt{\left(k_{1}+k_{-1}\right)^{2}+k_{-1}^{2}}}{k_{1}+k_{-1}} \xi_{2}(0) \\
& B(0)=-\xi_{1}(0)+\frac{k_{-1}}{k_{1}+k_{-1}} \xi_{2}(0)
\end{aligned}
$$

where $\xi_{1}(0)$ and $\xi_{2}(0)$ are the initial values of the extents.

The extents are given by weighted sums of two exponential components with two different characteristic times, a short time $1 /\left(k_{1}+k_{-1}\right)$ and a long time $\left(k_{1}+k_{-1}\right) / k_{1} k_{2}$. For the extent $\xi_{1}$, the prefactor $\frac{k_{-1}}{\sqrt{\left(k_{1}+k_{-1}\right)^{2}+k_{-1}^{2}}}$ associated with the long characteristic time is smaller than the absolute prefactor equal to 1 associated with the short characteristic time. Over the short time scale $t \leq 1 /\left(k_{1}+k_{-1}\right)$ the extent $\xi_{1}$ evolves as the fast exponential component provided that the initial conditions obey

$$
|A(0)| \leq|B(0)|
$$

The extent $\xi_{1}$ is considered as the fast variable.

Over the long time scale $t \gg 1 /\left(k_{1}+k_{-1}\right)$, the fast exponential decays in Eqs. $(23,24)$ are negligible leading to

$$
\begin{aligned}
& \xi_{1}(t) \simeq \frac{k_{-1}}{\sqrt{\left(k_{1}+k_{-1}\right)^{2}+k_{-1}^{2}}} A(0) \exp \left(-\frac{k_{1} k_{2}}{k_{1}+k_{-1}} t\right) \\
& \xi_{2}(t) \simeq \frac{k_{1}+k_{-1}}{\sqrt{\left(k_{1}+k_{-1}\right)^{2}+k_{-1}^{2}}} A(0) \exp \left(-\frac{k_{1} k_{2}}{k_{1}+k_{-1}} t\right)
\end{aligned}
$$

which amounts to

$$
\xi_{1}(t) \simeq \frac{k_{-1}}{k_{1}+k_{-1}} \xi_{2}(t)
$$

or, according to Eq. (21), to

$$
\frac{\mathrm{d} \xi_{1}}{\mathrm{~d} t}=0
$$


The partial-equilibrium approximation consists in considering that the fast exponential component instantaneously relaxes which amounts to eliminating the fast extent $\xi_{1}$. However the fast extent $\xi_{1}$ has not reached a steady state but it keeps evolving at the rate imposed by the extent $\xi_{2}$. In other words, the reaction associated with the fast extent $\xi_{1}$ adapts to the instantaneous value of the other extent $\xi_{2}$ : The fast extent reaches a pseudo-equilibrium state for each value of the slowly evolving extent as in an adiabatic process.

The elimination of the fast extent $\xi_{1}$ leads to the reduced reaction scheme

$$
\mathrm{Y} \stackrel{\stackrel{k_{1} k_{2}}{k_{1}+k_{-1}}}{\longrightarrow} \mathrm{Z}
$$

that needs to be supplemented by the relationship

$$
X=\frac{k_{-1}}{k_{1}} Y
$$

deduced from Eqs. (11,31).

\section{Elimination of a fast variable}

The quasi-steady-state approximation (QSSA) and the partial-equilibrium approximation (PEA) offer well-known methods to eliminate a fast variable, a concentration or an extent. ${ }^{15,18-22}$

These approximations are zeroth-order perturbation methods consisting in writing $\frac{\mathrm{d} v_{i}}{\mathrm{~d} t}=0$ for a fast variable $v_{i}$ in a system of differential equations involving $n$ variables. $^{4,5,23-27}$

After linearization of the system of differential equations around a stable steady state, standard linear algebra calculations give the expression of each variable in the form of a weighted sum of exponential decays. The weights depend on the rate constants and the initial conditions. In particular, the characteristic times are defined as the inverse of the absolute real part of the eigenvalues of the Jacobian matrix.

The elimination of the fast variable $v_{i}$ can be performed if two necessary conditions are fulfilled. ${ }^{5}$ First, one characteristic time $\tau_{s}$ must be much smaller than the others, leading to a fast exponential decay. Second, the weight of the fast exponential decay $\exp \left(-t / \tau_{s}\right)$ must be larger than or equal to the weight of each other exponential decay in the expression of the variable $v_{i}$. These two conditions involve the rate constants. In addition, the approximation is valid for appropriate initial conditions such that the variables have reached the so-called slow manifold of dimension $n-1^{14-16}$ defined by

$$
\frac{\mathrm{d} v_{i}}{\mathrm{~d} t}=0
$$


over the long time scale obeying $t \gg \tau_{s}$. On the slow manifold, the variable $v_{i}$ adapts to the evolution of the other variables and evolves at a rate imposed by them.

After the elimination of the fast variable $v_{i}$, the system of differential equations governing the evolution of the other variables can be written as a system of dimension $n-1$. If this system of differential equations for concentrations involves polynomials compatible with chemical kinetics rate laws, the evolution of the remaining chemical species can be associated with a reduced set of reaction steps. The existence of a reduced reaction scheme is not always guaranteed as illustrated by Michaelis-Menten kinetics which leads to rational functions of the concentrations in the rate equations. ${ }^{21}$ If the remaining characteristic times have different orders of magnitude, an analogous procedure of elimination may be performed to further reduce the number of variables.

\section{References}

${ }^{1}$ Miles S Okino and Michael L Mavrovouniotis. Simplification of mathematical models of chemical reaction systems. Chemical reviews, 98(2):391-408, 1998.

${ }^{2}$ SK Shoffner and Santiago Schnell. Approaches for the estimation of timescales in nonlinear dynamical systems: Timescale separation in enzyme kinetics as a case study. Mathematical biosciences, 287:122-129, 2017.

${ }^{3}$ Justin Eilertsen, Wylie Stroberg, and Santiago Schnell. Characteristic, completion or matching timescales? an analysis of temporary boundaries in enzyme kinetics. Journal of theoretical biology, 481:28-43, 2019.

${ }^{4}$ Gabriel Morgado, Bogdan Nowakowski, and Annie Lemarchand. Elimination of fast variables in stochastic nonlinear kinetics. Physical Chemistry Chemical Physics, 22(36):20801-20814, 2020.

${ }^{5}$ Agnes Pellissier-Tanon, Gabriel Morgado, Ludovic Jullien, and Annie Lemarchand. Quasi-steady-state and partial-equilibrium approximations in chemical kinetics: one stage beyond the elimination of a fast variable. ChemRxiv, preprint, 2021.

${ }^{6}$ Chong Wha Pyun. Steady-state and equilibrium approximations in chemical kinetics. Journal of Chemical Education, 48(3):194, 1971.

${ }^{7}$ Nicolaas Godfried Van Kampen. Elimination of fast variables. Physics Reports, 124(2):69-160, 1985.

${ }^{8}$ Alexander N Gorban and Iliya V Karlin. Method of invariant manifold for chemical kinetics. Chemical Engineering Science, 58(21):4751-4768, 2003. 
${ }^{9}$ Alexander N Gorban, Ovidiu Radulescu, and Andrei Y Zinovyev. Asymptotology of chemical reaction networks. Chemical Engineering Science, 65(7):2310-2324, 2010.

${ }^{10}$ Dimitris A Goussis. Quasi steady state and partial equilibrium approximations: their relation and their validity. Combustion Theory and Modelling, 16(5):869-926, 2012.

${ }^{11}$ Alexander N Gorban. Model reduction in chemical dynamics: slow invariant manifolds, singular perturbations, thermodynamic estimates, and analysis of reaction graph. Current Opinion in Chemical Engineering, 21:48-59, 2018.

${ }^{12}$ Margaret Rae and Mário N Berberan-Santos. Pre-equilibrium approximation in chemical and photophysical kinetics. Chemical physics, 280(3):283-293, 2002.

${ }^{13}$ Margaret Rae and Mário N Berberan-Santos. A generalized pre-equilibrium approximation in chemical and photophysical kinetics. Journal of chemical education, 81(3):436, 2004.

${ }^{14}$ Neil Fenichel. Geometric singular perturbation theory for ordinary differential equations. Journal of differential equations, 31(1):53-98, 1979.

${ }^{15}$ Simon J Fraser. The steady state and equilibrium approximations: A geometrical picture. The Journal of chemical physics, 88(8):4732-4738, 1988.

${ }^{16}$ Justin Eilertsen, Wylie Stroberg, and Santiago Schnell. Phase-plane geometries in coupled enzyme assays. Mathematical biosciences, 306:126-135, 2018.

${ }^{17}$ Hermann Haken. Synergetics: Introduction and advanced topics. Springer Science \& Business Media, 2013.

${ }^{18}$ Max Bodenstein. Eine theorie der photochemischen reaktionsgeschwindigkeiten. Zeitschrift für physikalische Chemie, 85(1):329-397, 1913.

${ }^{19} \mathrm{~T}$ Turanyi, A S Tomlin, and M J Pilling. On the error of the quasi-steady-state approximation. The Journal of Physical Chemistry, 97(1):163-172, 1993.

${ }^{20}$ Lee A Segel. On the validity of the steady state assumption of enzyme kinetics. Bulletin of mathematical biology, 50(6):579-593, 1988.

${ }^{21}$ Lee A Segel and Marshall Slemrod. The quasi-steady-state assumption: a case study in perturbation. SIAM review, 31(3):446-477, 1989.

${ }^{22}$ Leonor Michaelis and Maude L Menten. The kinetics of the inversion effect. Biochem. Z, 49:333-369, 1913. 
${ }^{23}$ Robert E O'malley. Singular perturbation methods for ordinary differential equations, volume 89. Springer, 1991.

${ }^{24}$ Lena Noethen and Sebastian Walcher. Tikhonov's theorem and quasi-steady state. Discrete Contin. Dyn. Syst. Ser. B, 16(3):945-961, 2011.

${ }^{25}$ Alexandra Goeke and Sebastian Walcher. A constructive approach to quasi-steady state reductions. Journal of mathematical chemistry, 52(10):2596-2626, 2014.

${ }^{26}$ Alexandra Goeke, Sebastian Walcher, and Eva Zerz. Determining "small parameters" for quasi-steady state. Journal of Differential Equations, 259(3):1149-1180, 2015.

${ }^{27}$ Justin Eilertsen and Santiago Schnell. The quasi-steady-state approximations revisited: Timescales, small parameters, singularities, and normal forms in enzyme kinetics. Mathematical biosciences, 325:108339, 2020. 